\title{
LES PHLÉBOTOMES AMÉRICAINS
}

\author{
Clef D'identification de 97 femelles Connues $(*)$
}

\section{Par H. FLOCH et E. ABONNENC}

1. - Aile avec la nervure transversale médiane loin de la base et très rapprochée de la première bifurcation de la $2^{\circ}$ longitudinale $\left(\mathrm{R}^{2}+3+4\right)$, ce qui donne un très petit gamma - genre Warileya (**)......W. W. phlebotomanica Hertig, 1942 (Pl. VII, fig. 95).

- Aile avec la nervure transversale médiare près de la base et loin de la première bifurcation de la $2^{\circ}$ longitudinale $\left(\mathrm{R}^{2+3+4}\right)$, ce qui donne un gamma relativement grand - genre Phlebotomus.. 2

2. - Fémurs postérieurs armés d'épines ou de denticules plus ou moins

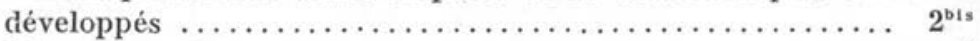

- Fémurs postérieurs inermes $\ldots \ldots \ldots \ldots \ldots \ldots \ldots \ldots \ldots$

$2^{\text {bis }}$ - Fémurs postérieurs présentant, sur la moitié proximale de la face interne, une rangée de 3 à 7 soies dont les bases d'insertion por-

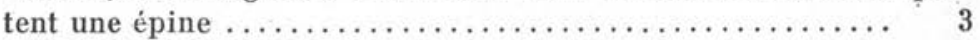

- Fémurs postérieurs présentant, sur la moitié de la face interne, une rangée de 13 fortes soies épineuses, courtes, spermathèques

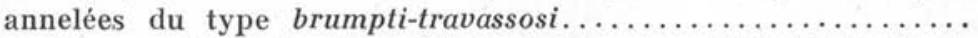
.P. spinosipes Floch et Abonnenc, 1943 (Pl. I, fig. 3).

3. - Pédoncule du corps de la spermathèque à parois épaisses, chitinisées, faisant suite à un conduit commun, sans conduit indivi-

(*) Les espèces rencontrées en Guyane ou aux Antilles françaises sont indiquées en caractères gras.

(**) G. B. Fairchild (Proc. Ent. Soc. Wash., Vol. LI, n² 2, 1949) a décrit, sous le nom de Hertigia hertigi (nouveau genre), une espèce qui appartiendrait à la sous-famille des Phlebotominz. Seul le mâle a été décrit, il présente un proboscis très développé et des maxilles dentés, ce qui fait suggérer à l'auteur que la femelle peut être hématophage.

La femelle n'étant pas connue, nous n'avons pas cru devoir faire figurer cette espèce dans notre clef, comme nous l'avons fait pour la seule connue du genre Warileya.

Le genre Hertigia peut aisément être séparé des genres Phlebotomus et Warileya par les caractères alaires suivants très marqués : ailes avec la nervure transverse médiane loin de la base, tandis que la première bifurcation de la $2^{\text {b longitudinale }\left(\mathrm{R}^{2}+3+4\right)}$ en est relativement rapprochée, ce qui donne un gamma négatif.

Ann, de Parasitologie, T. XXVI, $\mathrm{N}^{\circ \mathrm{s}} 1-2 .-1951$. 
duel ou par l'intermédiaire d'un conduit à peine ébauché ; longueur du conduit commun comparable à celle du pédoncule de la spermathèque......P. damascenoi Mangabeira, 1941 (Pl. I, fig. 1).

- Pédoncule du corps de la spermathèque séparé du conduit commun par un conduit individuel membraneux nettement mar-

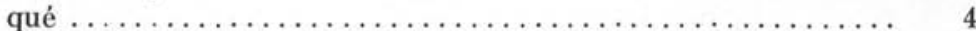

4. - Longueur totale des palpes supérieure à $800 \mu$; armature buccale constituée par 4 longues et fortes dents horizontales subégales, dirigées vers l'arrière, dont l'extrémité atteint ou dépasse le bord postérieur de la cavité, et plus en avant par une petite couronne

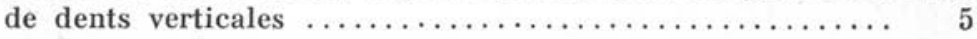

- Longueur totale des palpes inférieure à $700 \mu$; armature buccale constituée par une frange de nombreuses dents courtes, horizontales, irrégulières, dont les centrales sont légèrement plus fortes et plus longues que les latérales, et, parmi ces dernières, une vingtaine de petites dents verticales réparties régulièrement........ ........ P. spinosus Floch et Abonnenc, 1942 (Pl. I, fig. 2).

5. - Corps de la spermathèque globuleux, de 18 à $24 \mu$ de long pour $16 \mu$ de large, souvent séparé du pédoncule par une ligne de démarcation ; pédoncule fortement chitinisé.............. .......... p. pessoai Coutinho et Barretto, 1941 (Pl. I, fig. 4).

- Corps de la spermathèque presque cylindrique, de 29-32 $\mu$ de long pour $18-24 \mu$ de large, non séparé du pédoncule, qui n'est pas

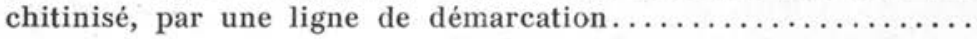
P. fischeri Pinto, 1926 (Pl. I, fig. 5).

6. $-5^{\text {e }}$ segment des palpes plus long que le $3^{\text {e } \ldots \ldots \ldots \ldots \ldots \ldots .} 22$

- $5^{\mathrm{e}}$ segment des palpes plus court que le $3^{\circ} \ldots \ldots \ldots \ldots \ldots \ldots \quad 7$

7. - Palpes avec le $\mathrm{III}^{e}$ article plus long que IV $+\mathrm{V}$; articles II + III habituellement égaux aux doubles de IV + V ; segments de la sper-

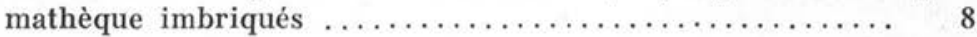

- Palpes avec le $\mathrm{III}^{\mathrm{e}^{-}}$article plus court que IV + V ; articles II + III plus courts que le double de IV $+\mathrm{V}$; corps de la spermathèque

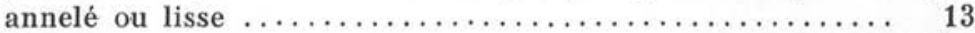

8. - Segments des palpes II + III plus de 3 fois aussi longs que IV $+\mathrm{V}$, conduits individuels plus courts que le corps de la spermathèque... P. sp. no 780 Floch et Chassignet, 1948 (Pl. I, fig. 9).

- Segments des palpes II + III plus de 3 fois aussi longs que IV $+\mathrm{V}$, conduits individuels 2 fois et demie aussi longs que le

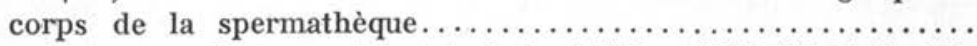
......... P. squamiventris Lutz et Neiva, 1912 (Pl. I, fig. 10).

- Segments des palpes II + III moins de 3 fois aussi longs que $\mathrm{IV}+\mathrm{V}$ 
9. - Conduits individuels de la spermathèque 2 fois aussi longs que le

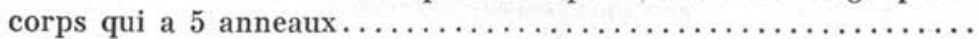

P. ayrozai Barretto et Coutinho, 1940 (Pl. I, fig. 8).

- Conduits individuels de la spermathèque plus de 2 fois aussi longs que le corps qui est composé de 8 segments ........... 10

- Conduits individuels de la spermathèque plus courts que le

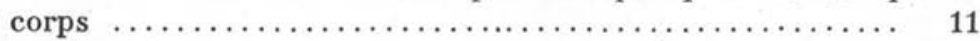

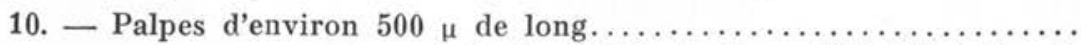
P. chagasi Costa Lima, 1941 (Pl. I, fig. 7).

- Palpes d'environ $650 \mu$ de long; longueur du conduit commun

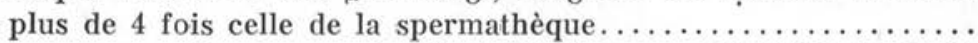

P. guyanensis Floch et Abonnenc, 1941 (Pl. I, fig. 6).

11. - Segments des palpes II + III ayant approximativement la longueur du double de IV $+\mathrm{V}$; corps de la spermathèque composé de 9 anneaux; cavité buccale munie de 2 paires de dents horizontales et d'une double rangée de fortes dents médianes ventrales, pas de plage pigmentée....... P. davisi Root, 1934 (Pl. I, fig. 11).

- Segments des palpes II + III distinctement plus longs que le dou-

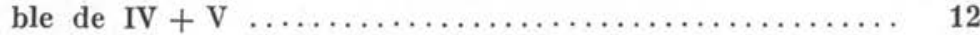

12. - Cavité buccale avec 4 paires de dents horizontales; dernier

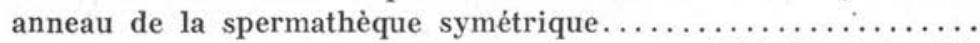
$\ldots \ldots \ldots \ldots \ldots \ldots$. arthuri Fonseca, 1936 (Pl. I, fig. 12).

- Cavité buccale avec 3 paires de dents horizontales; corps de la spermathèque composé d'environ 10 anneaux, dont le dernier est asymétrique........... P. lloydi Antunes, 1937 (Pl. I, fig 13).

- Cavité buccale avec 2 paires de dents horizontales et une double rangée de fortes dents médianes ventrales ; plage pigmentée présente ; corps de la spermathèque composé de 12 anneaux environ P. panamensis Shannon, 1926 (Pl. I, fig. 14).

- Cavité buccale avec 2 paires de dents horizontales ; toutes les dents ventrales petites; pas de plage pigmentée ; corps de la spermathè-

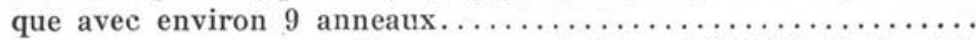
P. amazonensis Root, 1934 (Pl. II, fig. 15).

13. - Spermathèque de très petites dimensions n'ayant pas plus de $60 \mu$ (corps et conduits), toujours accompagnée d'une gaine chiti-

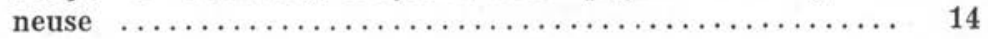

- Spermathèque bien développée (au moins $120 \mu$ de long, corps et conduits), non accompagnée d'une gaine chitineuse..... 15

14. - Corps de la spermathèque piriforme, émergeant d'une gaine chiti-

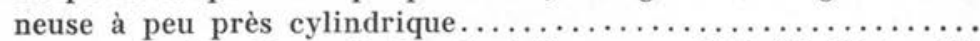
........ P. arborealis Floch et Abonnenc, 1944 (Pl. II, fig. 16).

- Corps de la spermathèque en massue grêle, enfermée avec son conduit dans une gaine chitineuse conique $\ldots \ldots \ldots \ldots \ldots \ldots$. 
P. sp. x. Floch et Abonnenc, 1944 (Pl. II, fig. 17).

15. - Conduits individuels et conduit commun de la spermathèque net-

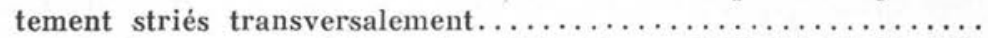
$\ldots \ldots \ldots \ldots \ldots \ldots$ P. anduzei Rozeboom, 1942 (Pl. II, fig. 19).

- Conduits individuels et conduit commun de la spermathèque non striés, lisses ou portant de petites saillies tuberculeuses.... 16

16. - Spermathèques présentant un conduit commun bien déve-

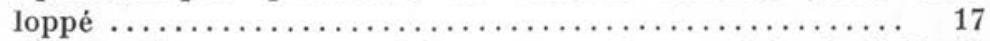

-- Spermathèques sans conduit commun, les conduits individuels aboutissant directement à l'orifice génital $\ldots \ldots \ldots \ldots \ldots \ldots 20$

17. - Conduit commun relativement long, plus de trois fois aussi long que les conduits individuels; corps de la spermathèque composé

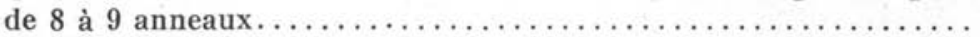

..... P. machicouensis Floch et Abonnenc, 1944 (Pl. II, fig. 20).

- Conduit commun moins de deux fois aussi long que les conduits individuels, parfois plus court que ces derniers ........ 18

18. - Corps de la spermathèque composé de 20-24 anneaux dont le ter-

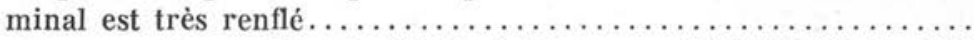
....... P. cauchensis Floch et Abounenc, 1943 (Pl. II, fig. 26).

- Corps de la spermathèque composé de 12 anneaux au maximum, le dernier n'étant pas manifestement renflé........... 19

19. - Corps de la spermathèque composé de 7 anneaux; rapport: conduit individuel

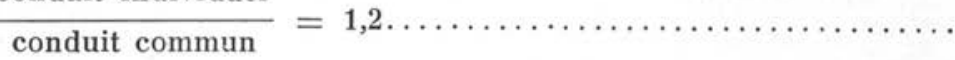

..... P. sp. de Souvenir Floch et Abonnenc, 1944 (Pl. II, fig. 21).

- Corps composé de 10 anneaux; rapport: $\frac{\text { conduit individuel }}{\text { conduit commun }}=0,68$

P. apicalis Floch et Abonnenc, 1943 (Pl. II, fig. 22).

20. - Conduits individuels très longs $(600 \mu)$; corps composé d'une trentaine de segments dont le terminal, très long et renflé, occupe

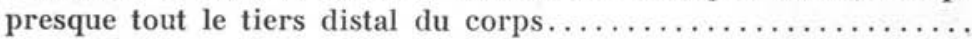
$\ldots \ldots \ldots \ldots \ldots$ P. ininii Floch et Abonnenc, 1943 (Pl. II, fig. 23).

- Conduit individuel ayant moins de $400 \mu$ de longueur ; corps composé d'une vingtaine de segments dont le terminal est à peine

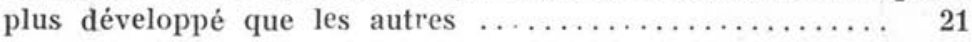

21. - Le corps de la spermathèque a les segments basaux de même largeur que le conduit individuel qui est au moins 5 fois aussi long

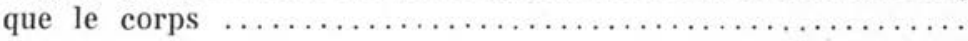

........ P. whitmani Antunes et Coutinho, 1939 (Pl. II, fig. 24). 
- Le corps de la spermathèque a les segments basaux plus larges que le conduit individuel qui n'est pas plus de 3 fois et demie

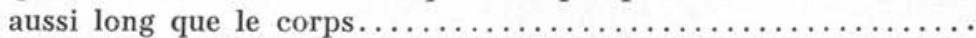

P. intermedius Lutz et Neiva, 1912 (Pl. II, fig. 25).

22. - Corps de la spermathèque terminé par une énorme expansion globuleuse qui enveloppe la tête et la déplace latéralement. Cette expansion représente environ 5 fois le volume du corps de la

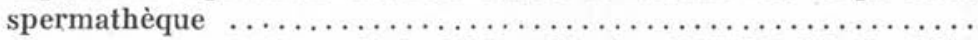

...... P. vesiciferus Fairchild et Hertig, 1947 (Pl. III, fig. 27) ;

...... P. deleoni Fairchild et Hertig, 1947 (Pl. III, fig. 28) ;

..... P. anthophorus Addis, 1945 (Pl. II, fig. 18).

- Corps de la spermathèque non terminé par une énorme expansion

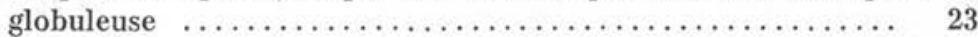

23. - Corps de la spermathèque, non compris le bouton terminal (tête), au moins 5 fois aussi long que sa largeur maximum ...... 24

- Corps de la spermathèque, non compris le bouton terminal (tête), plus de 3 fois, mais moins de 5 fois, aussi long que sa largeur

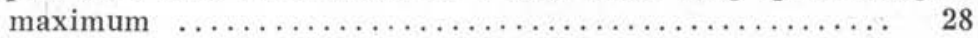

- Corps de la spermathèque, non compris le bouton terminal (tête), au moins 2 fois, mais pas plus de 3 fois, aussi long que sa largeur

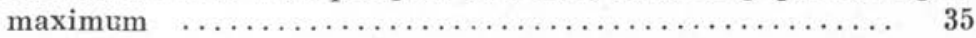

- Corps de la spermathèque, non compris le bouton terminal (tête), au moins aussi long que sa largeur maximum, mais pas plus d'une fois et demie cette largeur $\ldots \ldots \ldots \ldots \ldots \ldots \ldots \ldots \ldots \ldots .45$

- Corps de la spermathèque, non compris le bouton terminal (tête),

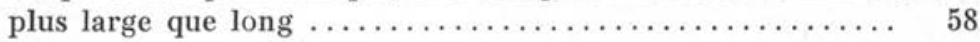

24. - Corps de la spermathèque spiralé (3-4 spires irrégulières) ; conduits individuels longs de $415 \mu$ environ; pas de conduit commun........ P. aragaoi Costa Lima, 1932 (Pl. III, fig. 29).

- Corps de la spermathèque aznelé ................ 25

- Corps de la spermathèque lisse $\ldots \ldots \ldots \ldots \ldots \ldots \ldots \ldots 26$

25. - Conduits individuels aussi larges que le corps de la spermathèque (en leur largeur maximum) et 8 à 9 fois plus longs que celui-ci ; $\mathrm{V}^{\mathrm{e}}$ segment des palpes plus court que les segments II + III. . . . . ........ P. fluviatilis Floch et Abonnenc, 1944 (Pl. IV, fig. 46).

- Conduits individuels très étroits et pas plus de 2 fois aussi longs que le corps de la spermathèque; $\mathrm{V}^{\mathrm{e}}$ segment des palpes plus long

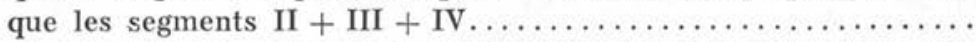

P. sp. de Saül Floch et Abonnenc, 1944 (Pl. III, fig. 30).

- $\mathrm{V}^{\circ}$ segment des palpes à peine plus long que le $\mathrm{III}^{\circ}$; spermathèques de 12-14 anneaux; épines géniculées avec prolongements postérieurs très longs, dépassant l'articulation sous-jacente...... .... P. driesbachi Causey et Damasceno, 1944 (Pl. III, fig. 31). 
26. - Corps de la spermathèque recourbé en forme de faux, présentant un bourrelet bien marqué à sa jonction avec le conduit individuel;

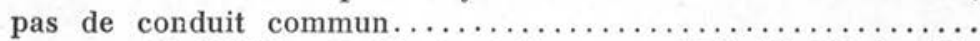
....... P. falciformis Floch et Abonnenc, 1944 (PI. III, fig. 32).

- Corps de la spermathèque non falciforme, faisant suite au conduit individuel sans démarcation brusque $\ldots \ldots \ldots \ldots \ldots \ldots .27$

27. - Corps de la spermathèque 10 fois aussi long que sa largeur maximum, se rétrécissant graduellement de l'apex à la base......

P. migonei França, 1920 (Pl. III, fig. 33).

- Corps de la spermathèque pas plus de 7 fois aussi long que sa largeur maximum, brusquement rétréci à sa base. Bucco-pharynx armé de dents fortes, très caractéristiques.................. ..... P. yucatanensis Galliard, 1931;

..... P. baduelensis Floch et Abonnenc, 1931 (Pl. III, fig. 34) ; $\ldots \ldots$ (=P. villelai Mangabeira, 1942).

28. - Cavité buccale armée de 22 à 24 dents horizontales fines, longues et aiguës, disposées en une seule rangée et munies chacune d'une arête centrale. Conduit individuel un peu plus de deux fois aussi long que le corps de la spermathèque.................... .............. P. chiapanensis, Dampf, 1947 (Pl. III, fig. 35).

- Cavité buccale armée de 4 à 10 dents horizontales disposées en une ou plusieurs rangées $\ldots \ldots \ldots \ldots \ldots \ldots \ldots \ldots \ldots . .29$

29. - Corps de la spermathèque en forme de carotte, diminuant progressivement de largeur de l'apex à la base, très nettement annelé sur toute sa longteur, le dernier anneau à peine plus déve-

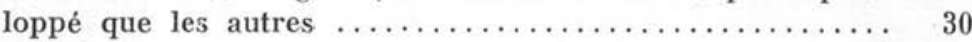

- Corps de la spermathèque plus ou moins cylindrique, fusiforme ou en forme de cigare, la partie apicale n'étant pas la plus

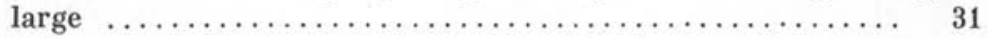

30. - Conduits individuels de la spermathèque très longs et filiformes, plus de 10 fois aussi longs que le corps de la spermathèque...... ..... P. avellari Costa Lima, 1932 (Pl. III, fig. 37) ;

..... P. guimaraesi Coutinho et Barretto, 1942 (Pl. III, fig. 38) ;

..... P. travassosi Mangabeira, 1942 (PI. III, fig. 36).

- Conduit individuel pas plus de 5 fois aussi long que la spermathèque. P. noguchii Shannon, 1939 (Pl. III, fig. 39) ;

P. osornoi Ristorcelli et Dâo Van Ty, 1941 (Pl. IV, fig. 40).

31. - Corps de la spermathèque cylindrique et nettement annelé, conduits individuels très courts, plus courts que le corps de la spermathèque et que le conduit commun $\ldots \ldots \ldots \ldots \ldots \ldots . .32$

- Corps de la spermathèque lisse ou strié plus ou moins fusiforme 
32. - Corps de la spermathèque avec 16 anneaux environ, le terminal

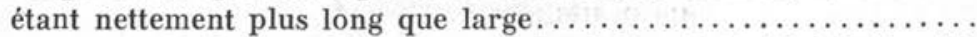
........ P. bourrouli Barretto et Coutinho, 1941 (Pl. IV, fig. 41).

- Corps de la spermathèque avec 24 anneaux environ, le terminal

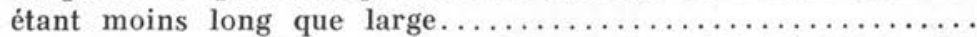

P. infraspinosus Mangabeira, 1941 (Pl. IV, fig. 42).

33. - Palpes longs, plus de $900 \mu$; corps de la spermathèque régulièrement fusiforme, plus de 4 fois aussi long que large $(4,5)$ dans sa plus grande largeur ; rapport : $\frac{\text { conduit commun }}{\text { corps de la spermathèque }}=6,1$. P. pacæ Floch et Abonnenc, 1943 (Pl. IV, fig. 43).

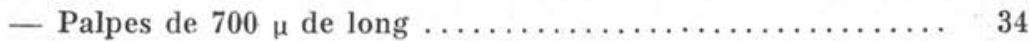

34. - Conduit individuel plus court que le corps de la spermathèque ; rapport : $\frac{\text { conduit commun }}{\text { corps de la spermathèque }}=2,3 \ldots \ldots \ldots \ldots \ldots \ldots \ldots$ P. shannoni Dyar, 1929 (Pl. IV, fig. 45) ;

............. (= limai Fonseca, 1935) ; $\ldots \ldots \ldots \ldots \ldots . \quad(=$ P. bigeniculatus Floch et Abonnenc, 1941).

-- Conduit individuel beaucoup plus long que le corps ce la spermathèque qui est dépourvu de bouton terminal; $\mathrm{III}^{\mathrm{e}}$ segment des

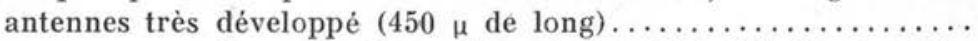
P. nordestinus Mangabeira, 1942 (Pl. IV, fig. 44) ; $\ldots \ldots \ldots \ldots{ }_{(=P}$. longicornutus, Floch et Abonnenc, 1943).

35. - Corps de la spermathèque nettement segmenté sur toute sa lon-

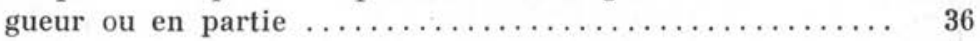

- - Corps de la spermathèque lisse, strié ou présentant seulement des bourrelets irréguliers ne formant pas d'anneaux bien définis 43

36. - Corps de la spermathèque nettement cylindrique, 9 anneaux bien définis........ P. longipalpis Lutz et Neiva, 1912 (Pl. IV, fig. 47).

-- Corps de la spermathèque diminuant régulièrement de largeur de l'apex à la base, la partie apicale étant la plus large..... 37

37. -- 6 anneaux subégaux, réguliers, répartis sur tout le corps de la spermathèque.... P. singularis Costa Lima, 1932 (Pl. IV, fig. 48).

-- Plus de 6 anneaux ou crénelures; s'il y en a 6 ou moins de 6 , le

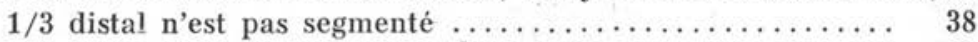

38. - - Ve segment des palpes plus long que les segments II + III + IV. . P. rorotaensis Floch et Abonnenc, 1944 (Pl. IV, fig. 49).

- Ve segment des palpes nettement plus court que les segments

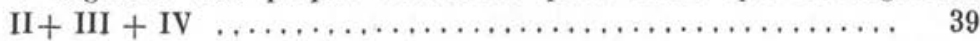


39. - V $\mathrm{V}^{\circ}$ segment des palpes plus court que les segments II + III ; rapconduits individuels

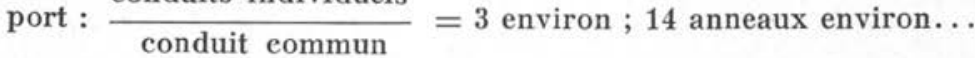

$\ldots \ldots \ldots \ldots \ldots$. lanei Barretto et Coutinho, 1941 (Pl. IV, fig. 50).
Vo segment des palpes plus long que les segments II + III... 40

40. - Dernier anneau de la spermathèque court, discoïdal..........

P. cavernicolus Costa Lima, 1932 (Pl. IV, fig. 54).

- Dernier anneau de la spermathèque long, globuleux ........

41. - Armature buccale constituée par 4 fortes dents horizontales subégales $\ldots \ldots \ldots \ldots \ldots \ldots \ldots \ldots \ldots \ldots \ldots \ldots \ldots \ldots \ldots \ldots \ldots \ldots \ldots, 42$

- Armature buccale constituée par 18-20 dents longues, fines et aiguës, munies chacune d'une arête centrale ; conduits individuels de la spermathèque pas plus de 3 fois aussi longs que le corps....

P. cayennensis Floch et Abonnenc, 1941 (Pl. IV, fig. 51).

- Armature buccale constituće par 34 dents horizontales, fines, longues et aiguës, munies chacune d'une arête centrale...........

P. ctenidophorus Fairchild et Hertig, 1948 (Pl. IV, fig. 52).

- Armature buccale constituée par 11 dents horizontales, fines, longues et aiguës, munies chacune d'une arête centrale; conduits individuels de la spermathèque 4 fois aussi longs que le corps.... ....P. cayennensis, var. puertoricensis, Fairchild et Hertig, 1948

(Pl. IV, fig. 53).

- Armature buccale constituée par 12-14 dents horizontales, fines, longues et aiguës, munies chacune d'une arête centrale.......... .... P. cayennensis, var. viequescensis, Fairchild et Hertig, 1948 (Pl. V, fig. 56) ; P. cayennensis, var. maciasi, Fairchild et Hertig, 1948 (PI. V, fig. 55).

42. $-\alpha / \beta=1,3$; plage pigmentée de la cavité buccale longue et étroite. ....... P. stewarti Mangabeira et Galindo, 1944 (Pl. V, fig. 57).

$-\alpha / \beta=2$; pas de plage pigmentée visible, conduits de la spermathèque 10 fois aussi longs que le corps de celle-ci .............. ............... P. gomezi Nitzulescu, 1931 (Pl. V, fig. 58) ; ................ (= . suis Rozeboom, 1940).

$-\alpha / \beta=2,6$; plage pigmentée peu marquée mais bien définie et bien visible; conduits de la spermathèque 6 fois aussi longs que le

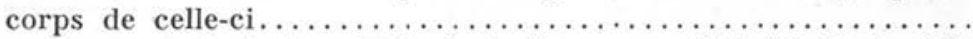

P. japignyi Floch et Abonnenc, 1944 (Pl. V, fig. 59).

43. - Corps de la spermathèque entièrement lisse, fusiforme, à parois épaisses ; conduit individuel + conduit commun plus de 3 fois aussi long que le corps de la spermathèque ..................

.. P. punctigeniculatus Floch et Abonnenc, 1944 (Pl. V, fig. 63). 
- Corps de la spermathèque cylindrique à extrémité arrondie ; pas de conduit commun; conduit individuel une fois et demie aussi

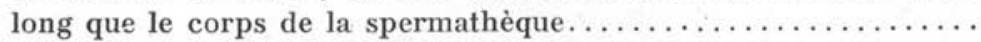
...... P. sp. de Maripa Floch et Abonnenc, 1946 (Pl. V, fig. 62).

- Corps de la spermathèque strié ou présentant des bourrelets irréguliers

44. - Corps de la spermathèque en forme de baril, strié dans le sens longitudinal; un bouton terminal bien développé ; conduits individuels très courts, plus courts que le corps de la spermathèque. . P. pestanai Barretto et Coutinho, 1941 (Pl. V, fig. 61).

- Corps de la spermathèque cylindrique et grossièrement segmenté sur toute sa longueur; conduits individuels très longs, plus de 7 fois la largeur du corps de la spermathèque.............. ................. P. almazani Galliard, 1934 (Pl. V, fig. 60).

- Corps de la spermathèque en forme de grosse massue, largeur $75 \mu$ environ, pas de bouton terminal visible, conduits individuels plus

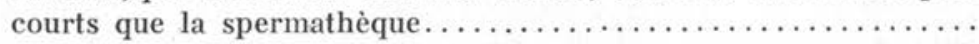
....... P. bursiformis Floch et Abonnenc, 1944 (Pl. V, fig. 65).

- Corps de la spermathèque grossièrement plissé, les anneaux mal définis, sauf l'apical qui est plus long et moins large que les autres; conduits individuels environ 2 fois aussi longs que le corps de la spermathèque, débouchant dans un conduit commun très large et un peu plus long que les conduits individuels...... ............ P. verrucarum Townsend, 1913 (Pl. V, fig. 66).

- Corps de la spermathèque en forme de carotte, finement strié, conduits individuels de même longueur que le corps de la spermathèque, conduit commun 2 fois plus long que celle-ci.......... .. P. columbianus Ristorcelli et Dâo Van Ty, 1941 (PI. V, fig. 67) ; . (= P. monticolus var. incarum Ristorcelli et Dâo Van Ty, 1941).

- Corps de la spermathèque conique, finement strié transversalement sur toute sa longueur, bouton terminal large et profondément invaginé dans le corps de la spermathèque ; conduits individuels très longs, 8 fois aussi longs que le corps de la spermathèque,

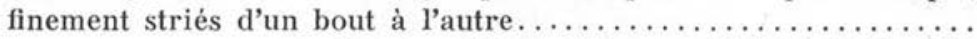
.............. P. evansi Nunez Tovar, 1924 (Pl. V, fig. 68).

45. - Corps de ìa spermathèque nettement annelé ou à bords cré-

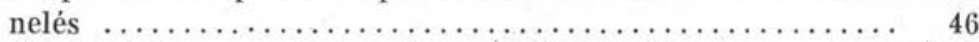

- Corps de la spermathèque lisse ou finement strié, ou quelquefois grossièrement plissé, sans anneaux nettement définis...... 48

46. - Ve segment des palpes plus court que les segments III + IV ; corps de la spermathèque en forme de poire ; 6 anneaux de même épaisseur répartis sur toute la longueur du corps................ ........... P. monticolus Costa Lima, 1932 (Pl. V, fig. 64).

- $V^{e}$ segment des palpes plus long que les segments III + IV.. 
47. - Corps de la spermathèque cylindrique avec 8-9 crénelures ; dernier anneau beaucoup plus large que les autres, pas de bouton terminal visible; conduits individuels très fins................ ......... P. amarali Barretto et Coutinho, 1940 (Pl. VI, fig. 69).

- Corps de la spermathèque composé de 4 gros anneaux dont les 2 médians sont moins larges et moins longs que l'apical et le basal; bouton terminal bien développé ; indice alaire $\alpha / \beta=5 \ldots$. P. alphabcticus Fonseca, 1936 (Pl. VI, fig. 70).

- Corps de la spermathèque conique, strié transversalement sur toute sa longueur; bouton terminal bien développé; conduits individuels longs, 6 fois environ la longueur du corps de la spermathèque; $\mathrm{V}^{0}$ segment des palpes aussi long que les segments $\mathrm{II}+\mathrm{III}+\mathrm{IV}$. . P. lichyi Floch et Abonnenc, 1950 (Pl. VI, fig. 71).

- Corps de la spermathèque piriforme, 5 à 7 anneaux répartis irrégulièrement sur la moitié basale du corps, la moitié apicale n'étant pas annelée, bouton terminal bien développé; conduits individuels de la spermathèque épais ; largeur du corps/largeur du conduit $=3$ environ ; indice alaire $\alpha / \beta=1,5 \ldots \ldots \ldots \ldots \ldots$.

P. oswaldoi Mangabeira, 1942 (Pl. VI, fig. 72).

48. - Corps de la spermathèque sans bouton terminal, globuleux, avec des soies fines, éparses sur la calotte apicale. Conduits individuels 5 fois aussi longs que le corps de la spermathèque.........

P. heckenrothi Floch et Abonnenc, 1942 (Pl. VI, fig. 73).

- Corps de la spermathèque sans bouton terminal globuleux. Conduit individuel très court, plus court que le corps de la spermathèque. Epines géniculées des antennes avec prolongements

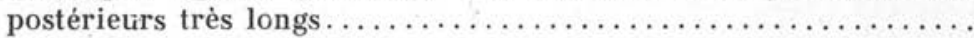

P. sp. no 768 Floch et Chassignet, 1948 (Pl. VI, fig. 74).

- Corps de la spermathèque muni d'un bouton terminal ou si celui-ci est peu visible, les soies émergent groupćes en bouquet de l'apex

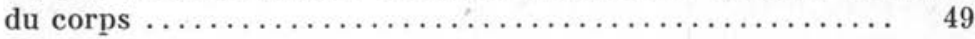

49. - $\mathrm{V}^{\circ}$ segment des palpes aussi long ou plus long que les segments

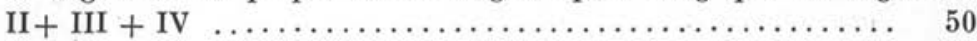

- $\mathrm{V}^{\circ}$ segment des palpes nettement plus court que les segments $\mathrm{II}+\mathrm{III}+$ IV

50. - Corps de la spermathèque en forme dè fourneau de pipe ; pas de séparation nette entre le corps et le conduit, si ce n'est un coude

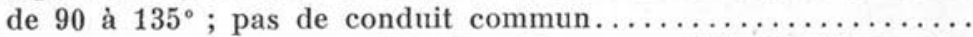

P. chassigneti Floch et Abonnenc, 1944 (Pl. VI, fig. 75).

-_ Corps de la spermathèque en forme de poire, nettement séparé du conduit individuel; conduit commun relativement long; cavité buccale armée de 4 dents horizontales très courtes, peu marquées ; plage pigmentée large et très assombrie.................. 
P. atroclavatus Knab, 1913 (Pl. VI, fig. 76) ;

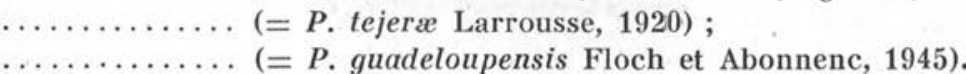

- Corps de la spermathèque en forme de poire nettement séparé du conduit individuel; cavité buccale armée de 4 dents larges et fortes bien visibles; plage pigmentée étroite et peu marquée..... ........ . zuliaensis Floch et Abonnenc, 1948 (Pl. VI, fig. 77).

51. - Corps de la spermathèque présentant sur sa moitié apicale de nombreux tubercules diminuant de grosseur de la base à l'apex. . ........ P. pinealis Floch et Abonnenc, 1944 (Pl. VI, fig. 78).

- Corps de la spermathèque lisse, finement strié ou plissé... 52

52. - Conduits individuels nettement plus courts que le conduit

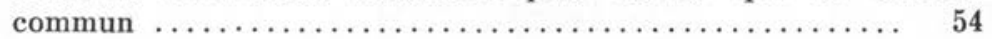

- Conduits individuels plus longs que le conduit commun... 56

— Pas de conduit commun; corps de la spermathèque globuleux $\quad 53$

53. - Conduit individuel 3 fois aussi long que le corps de la spermathèque ; 19 dents horizontales et 3 rangées (de 14 à 17 chacune) de

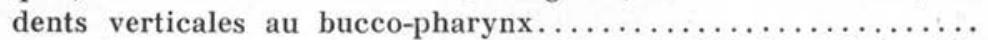
................ texanus Dampf, 1946 (Pl. VI, fig. 79).

- Conduits individuels au moins 6 fois aussi longs que le corps de la spermathèque ; 6 dents horizontales au bucco-pharynx....... P. trichopygus Floch et Abonnenc, 1945 (Pl. VI, fig. 80).

54. - Corps de la spermathèque se rétrécissant progressivement pour s'unir au conduit individuel qui est très court; rapport : conduit commun/conduit individuel $=3 \ldots \ldots \ldots \ldots \ldots \ldots \ldots \ldots \ldots \ldots \ldots$ ..... P. vespertilionis Fairchild et Hertig, 1947 (Pl. VII, fig. 81).

- Corps de la spermathèque brusquement rétréci à son union avec

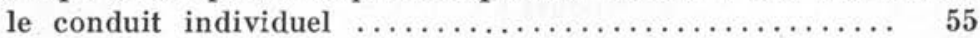

55. - Rapport : conduit commun/conduit individuel $=4,2$; corps de la spermathèque grossièrement cylindrique ; épines géniculées des

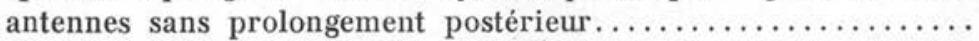
$\ldots \ldots \ldots \ldots \ldots$ P. choti Floch et Abonnenc, 1941 (Pl. VII, fig. 93).

- Rapport : conduit commun/conduit individuel $=2$ environ ; corps de la spermathèque grossièrement cylindrique ; épines géniculées

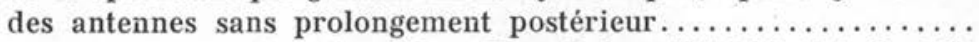

P. triacanthus Mangabeira, 1942 (Pl. VII, fig. 82).

- Rapport : conduit commun/conduit individuel $=2$ environ ; corps de la spermathèque globuleux; épines géniculées des antennes présentant des prolongements postérieurs aussi longs que la largeur du segment sur lequel elles sont insérées.............. ... P. sp. de Cayenne Floch et Abonnenc, 1945 (Pl. VII, fig. 83) ; ... (= P. lutzianus $?)$. 
56. - Rapport : conduit individuel/conduit commun $=1,1$; corps de la spermathèque en forme de carotte courte, diminuant régulièrement de largeur de l'apex à la base, finement strié transversalement........ P. longispinus Mangabeira, 1942 (Pl. VII, fig. 84).

- Rapport : conduit individuel/conduit commun supérieur à 2; corps de la spermathèque lisse, globuleux ou en forme de capu-

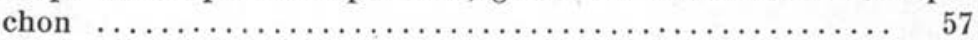

57. - Corps de la spermathèque globuleux, avec un col basal, rappelant une ampoule électrique ; bouton terminal court et arrondi....... ............. P. fonsecai Costa Lima, 1932 (Pl. VII, fig. 85).

- Corps de la spermathèque en forme de capuchon, plus large à la base qu'à l'apex; appendice terminal (tête) très allongé, fin et

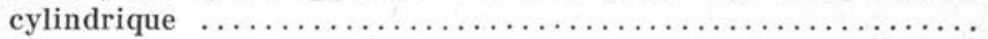
..... P. evandroi Costa Lima et Antunes, 1936 (Pl. VII, fig. 86).

58. - Corps de la spermathèque à peu près aussi large que le conduit

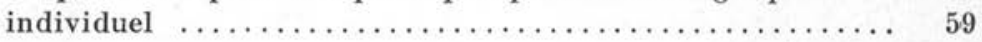

- Corps de la spermathèque nettement plus large que le conduit

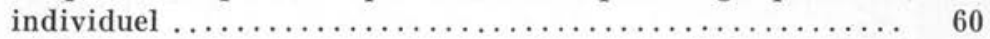

59. - Corps de la spermathèque petit, en forme de sphère aplatie de haut en bas, conduits individuels très longs, environ $600 \mu \ldots \ldots$. P. oppidanus Dampf, 1944 (Pl. VII, fig. 87).

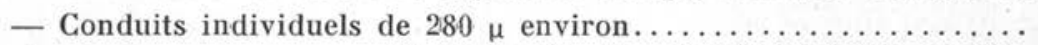
P. vindicator Dampf, 1944 (Pl. VII, fig. 88).

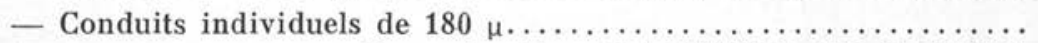
........... vexator Coquillet, 1907 (Pl. VII, fig. 88bis).

- Corps de la spermathèque en forme de capuchon, conduits individuels très larges à la base et diminuant progressivement de largeur de la base à l'apex, $140 \mu$ de longueur............... ..... P. sp. de Baduel Floch et Abonnenc, 1945 (Pl. VII, fig. 92).

60. - $\mathrm{V}^{e}$ segment des palpes plus long que les segments II + III...... ................ walkeri Newstead, 1914 (Pl. VII, fig. 89).

- Ve segment des palpes plus court que les segments II + III ..... .... P. cortelezzii Brethes, 1923 (Pl. VII, fig. 91);

... P. sallesi Galvâo, Ayroza et Coutinho, 1939 (Pl. VII, fig. 90) ;

.... P. edwardsi Mangabeira, 1941 (Pl. VII, fig. 94).

Institut Pasteur de la Guyane Française. 

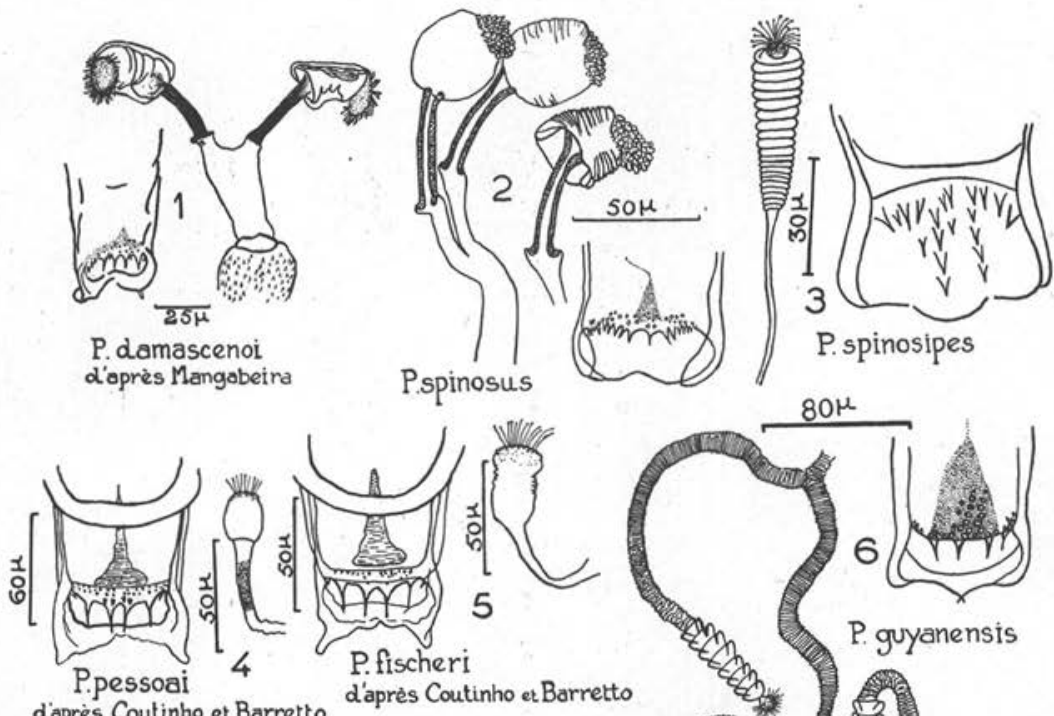
d'aprés Coutinho et Barretto

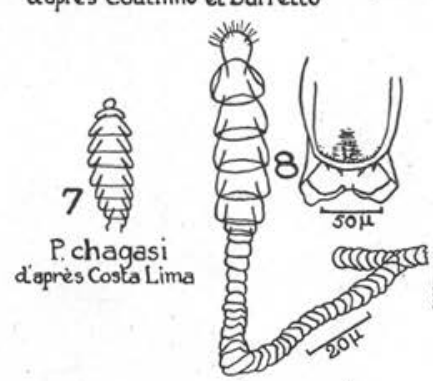

Payrozai

diopres Barretto et

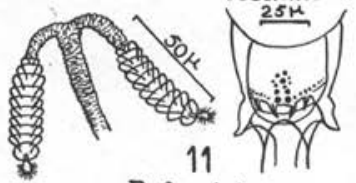

Al daprés Root
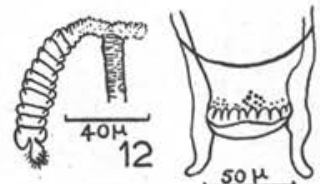

Parthuri spermath. d'aprés Galvào et Couthino

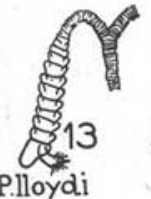
daaprès Galvao et Couthino

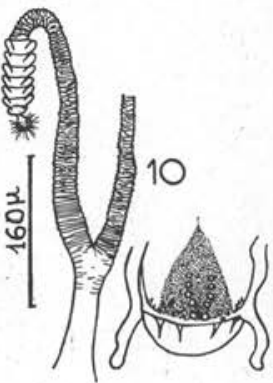

P. squamiventris

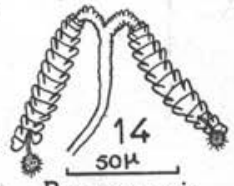

P. panamensis dapres Root

Planche I 

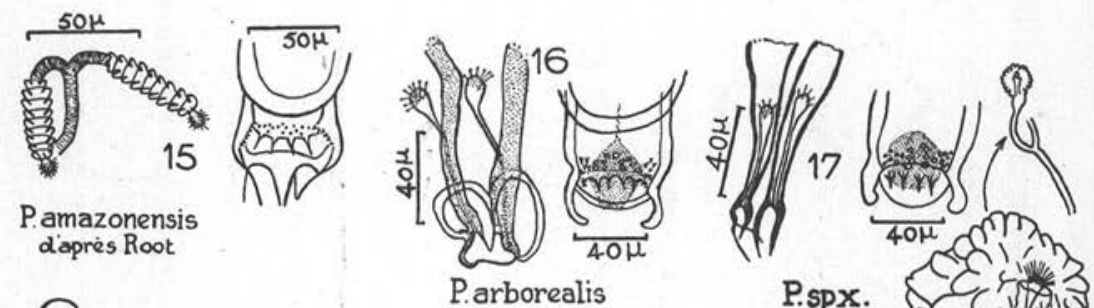

dopres Root

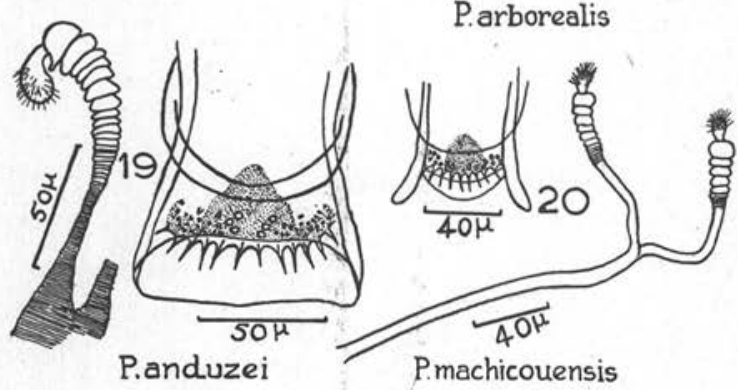

P.spx.

Eह द्
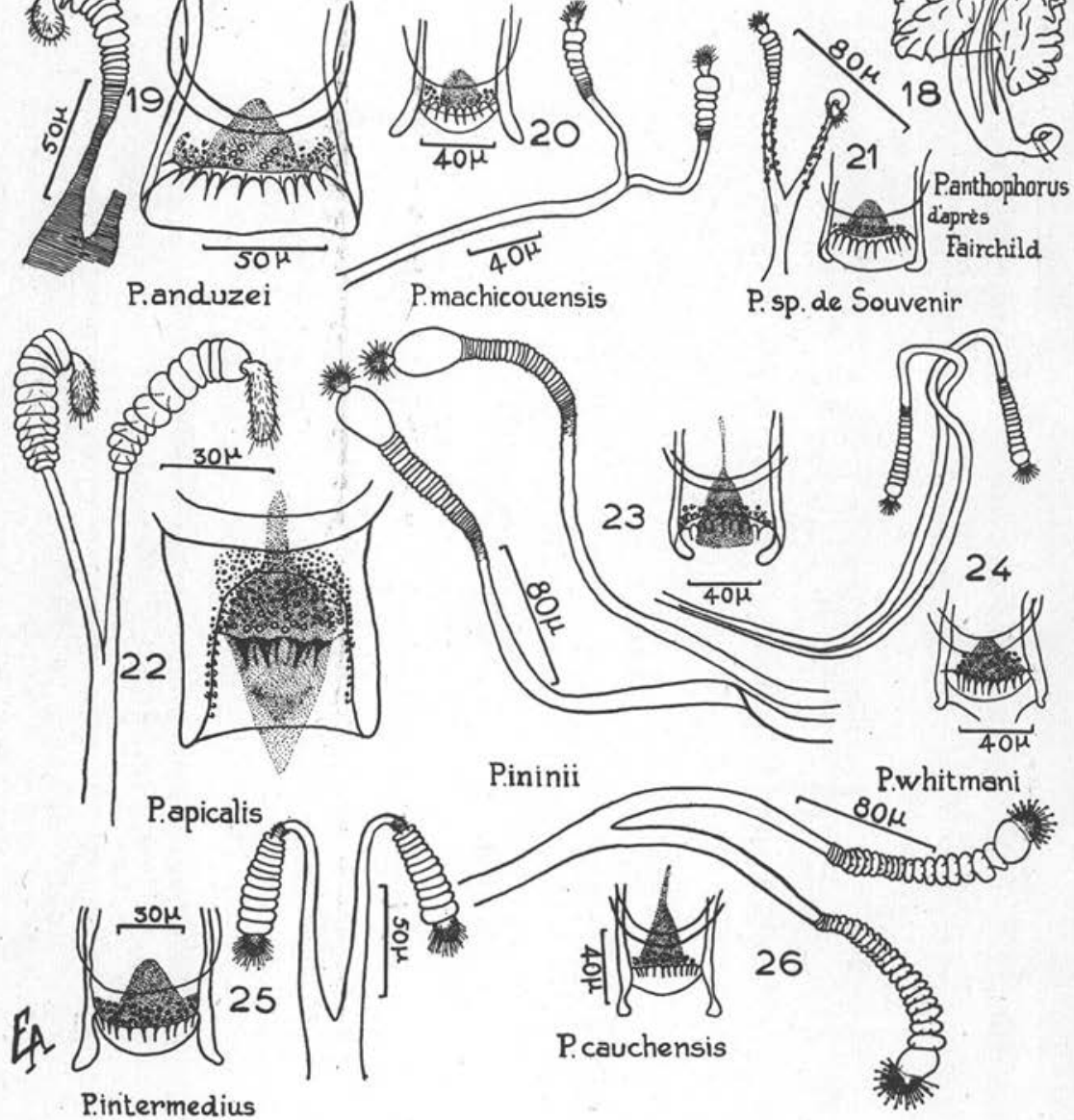

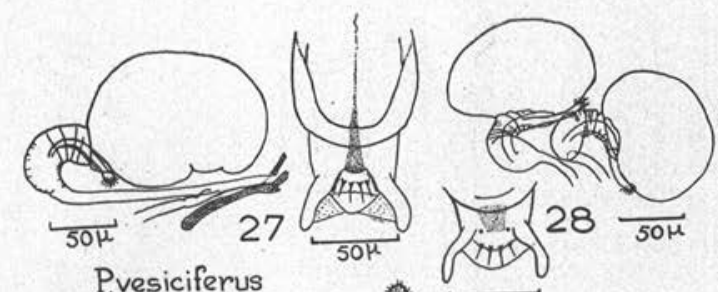

P.vesiciferus

d'après Fairchild et Hertig

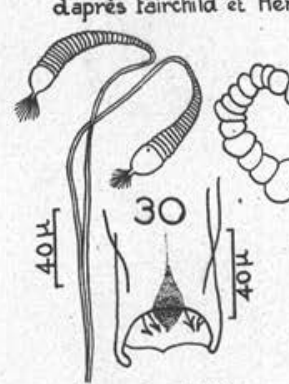

finos

50\%

P.deleoni
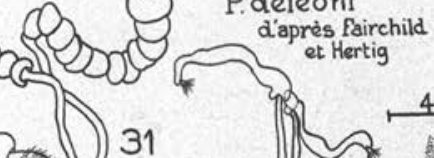

daprès fairchild
et Hertig
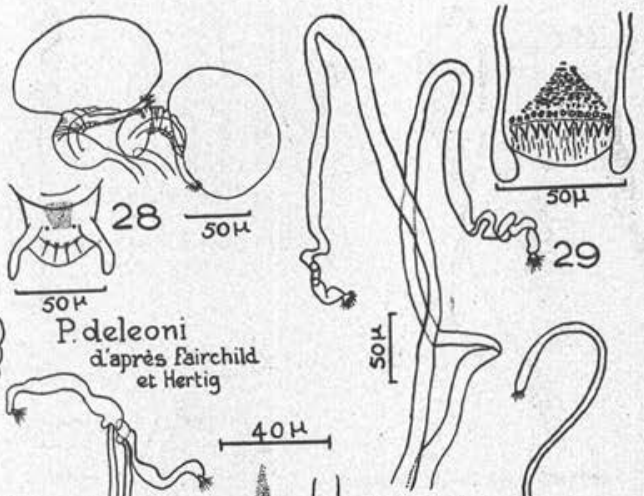

Psp. de Saül
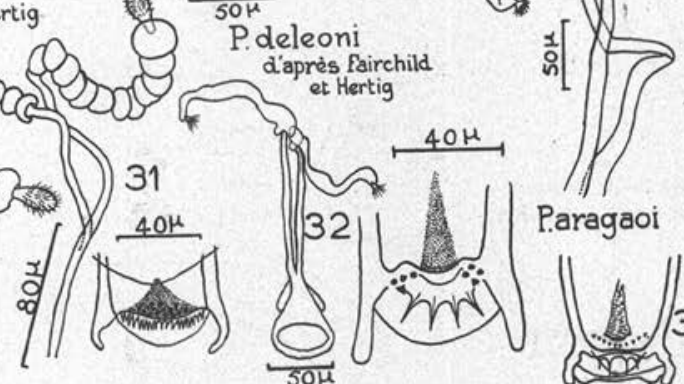

Paragaoi
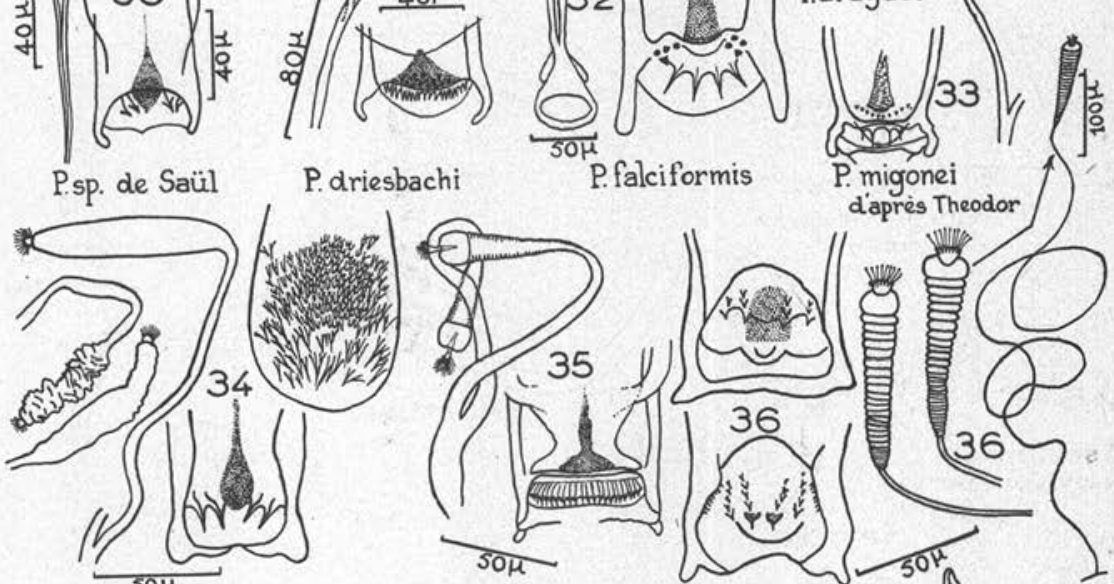

so $\mu$

P. baduelensis

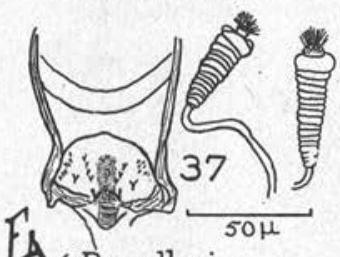

A) P.avellari

d'aprés Mangabeira

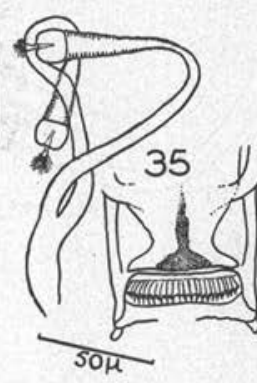

P.falciformis
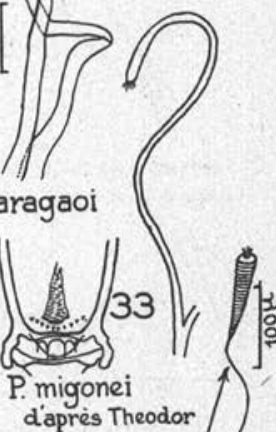

dapres Theodor

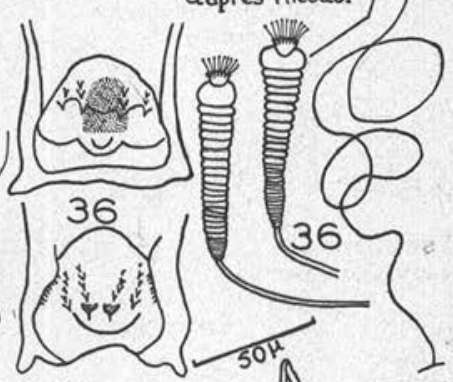

P.chiapanensis

daprés Fairchild et Hertig

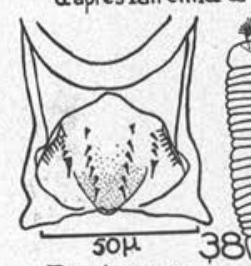

P.guimaraesi

d'apres Coutinho

P.travassosi

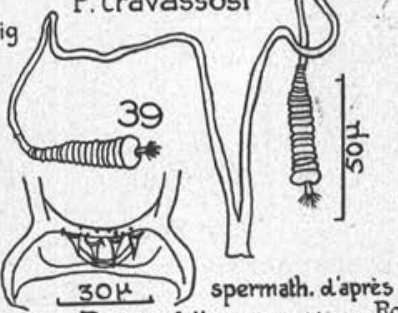

P.noguchii d'après Hertig Rozeboom

Planche III

Ann. de Parasitologie, T. XXVI, $\mathrm{N}^{\circ \mathrm{s}} 1-2 .-1951$.

8. 


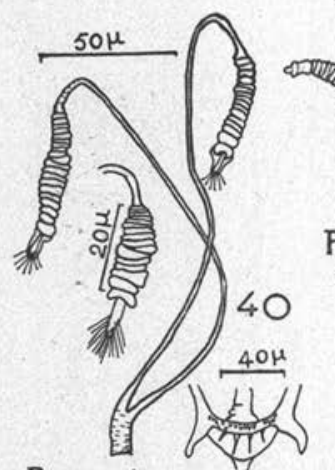

Posornoi daprès Rozeboom et d'après Ristorcelli et Van Ty

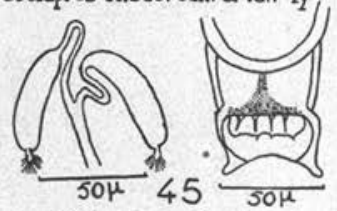

P. shannoni d'après

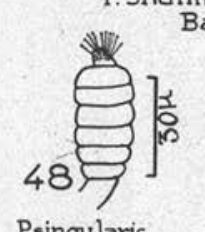
Psingularis
d'apres CostaLima

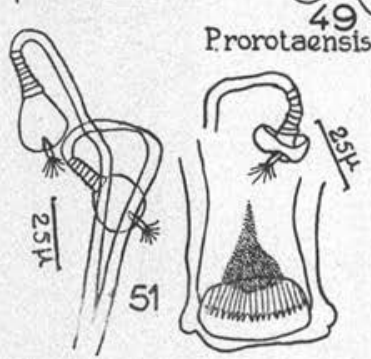

If Pcayennensis
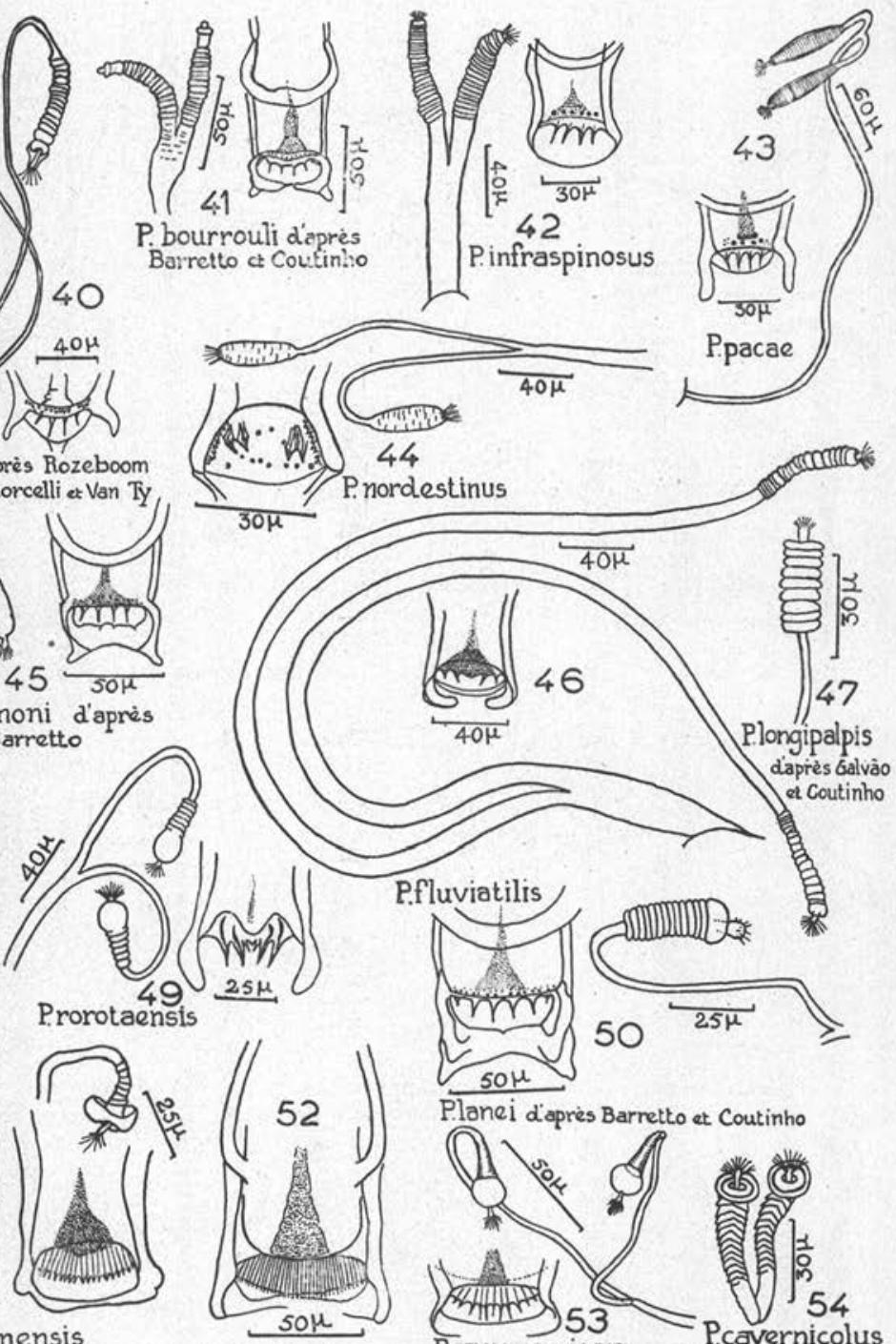

P.ctenidophorus daprés Earrehild é Hertig puertoricensis

dapres Fairchild etHertig 


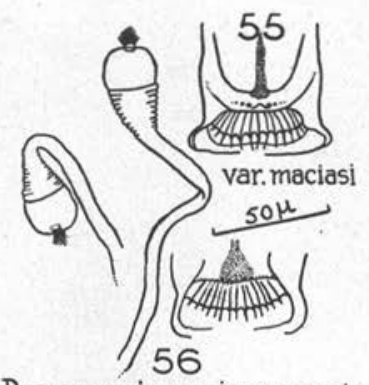

P.cayennensis var. viequescensis d'apres Fairchild et Hertig

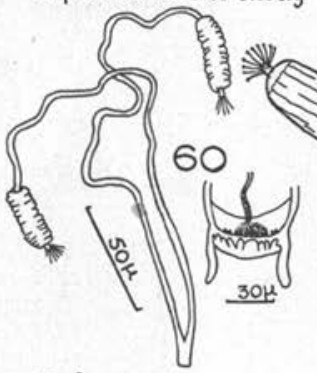

\section{P.almazani dapres} Dampf

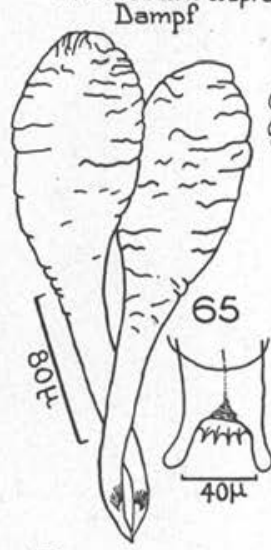

P.bursiformis

EA

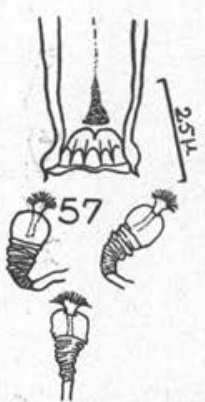

\section{Pstewarti}

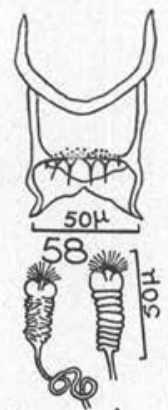

P.gomezi et Galindo

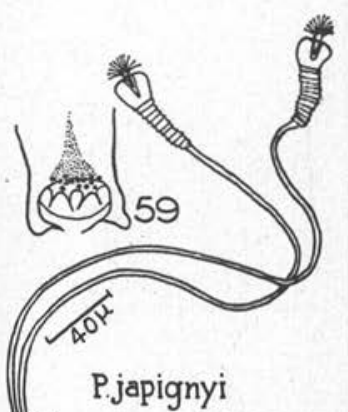

62

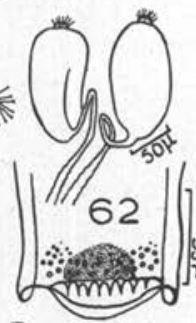

P.sp. de Maripa -culatus

P.pestanai

d'apres Coutinho et Barretto
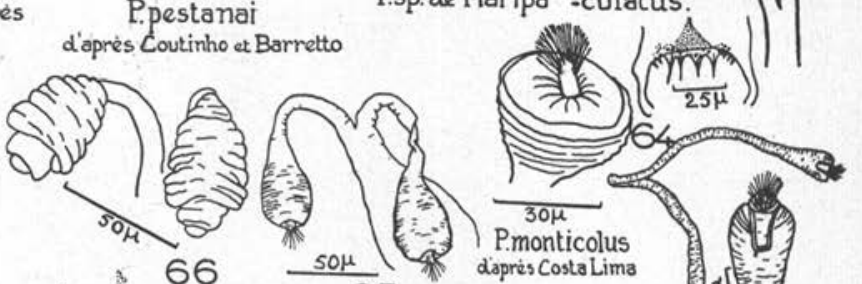

P.monticolus

67

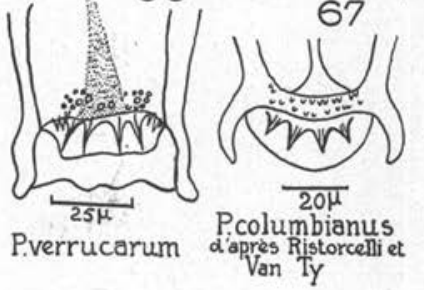

P.verrucarum

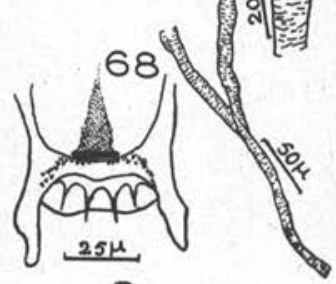

Pevansi

Planche V 


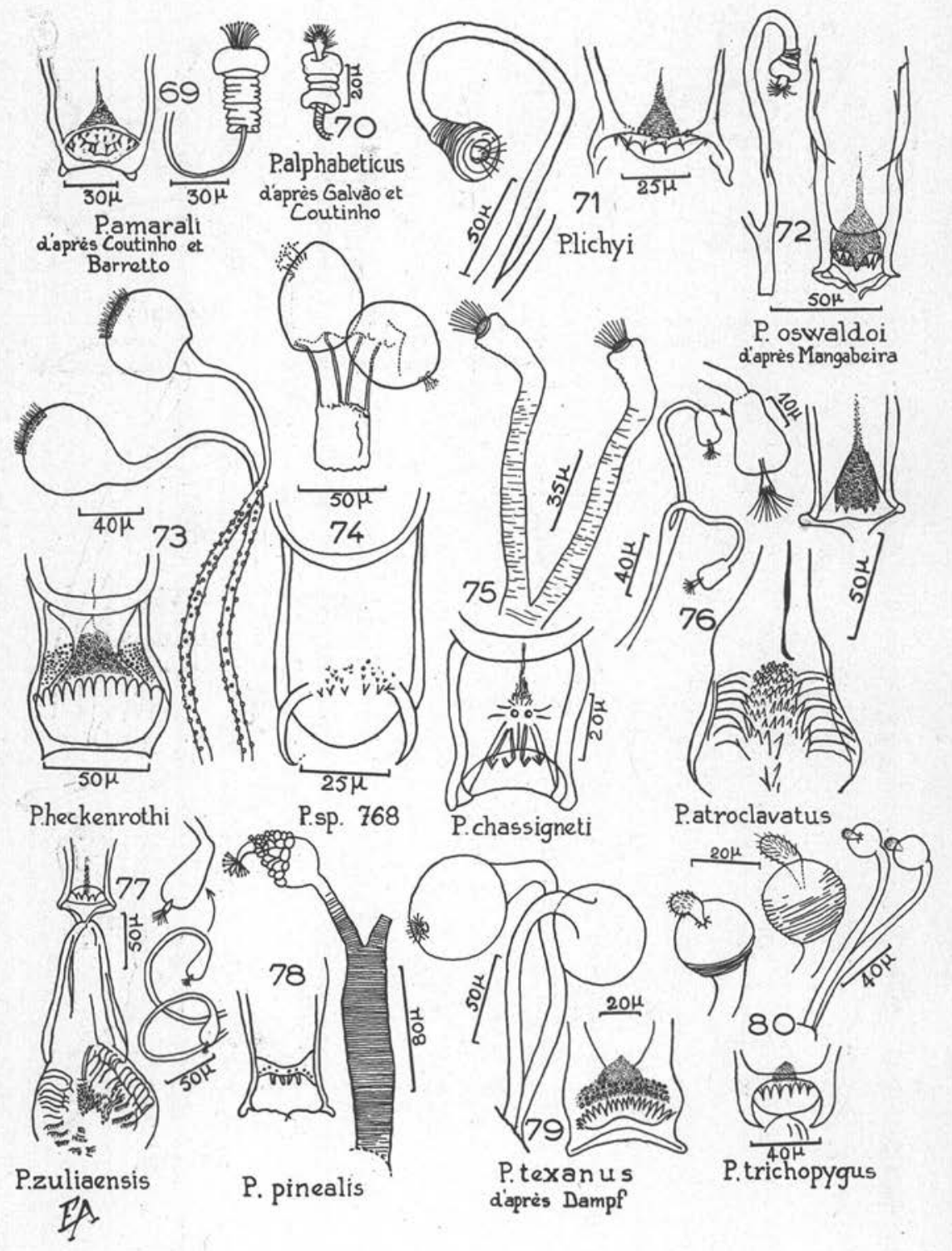

Planche, VI 


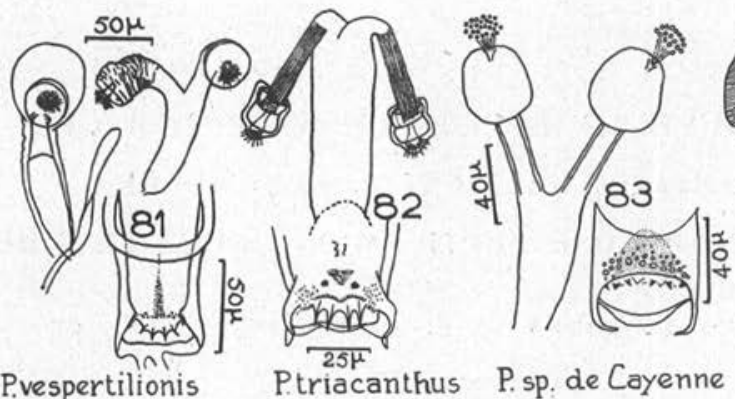

P.vespertilionis

Ptriacanthus

P. sp. de Cayenne

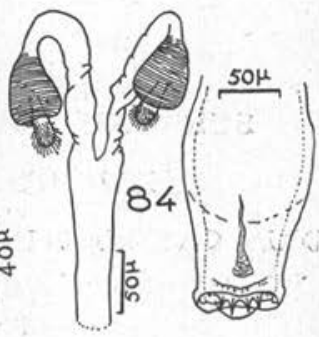

d'après Fairchild et Hertig d'après Mangabeira

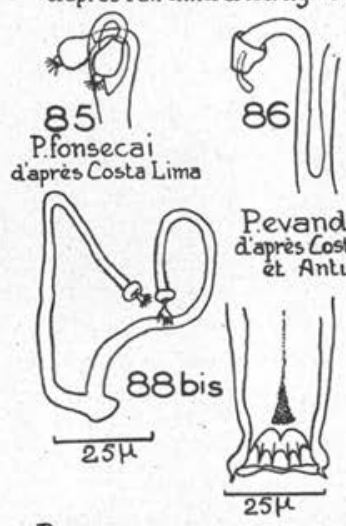

P. vexator diaprés Mangabeira

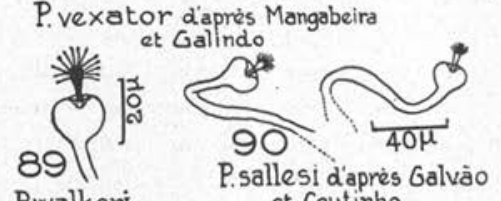

Pwalkeri

dapres Costa et Coutinho

Lima

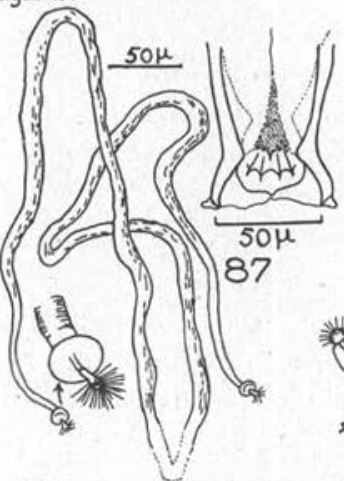

P.oppidanus dapres Dampf

P. longispinus daprès

Mangabeira

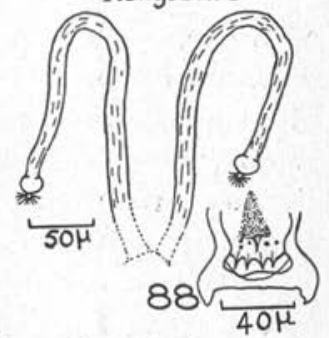

2 Pvindicator

$\checkmark$ diaprés Dampf
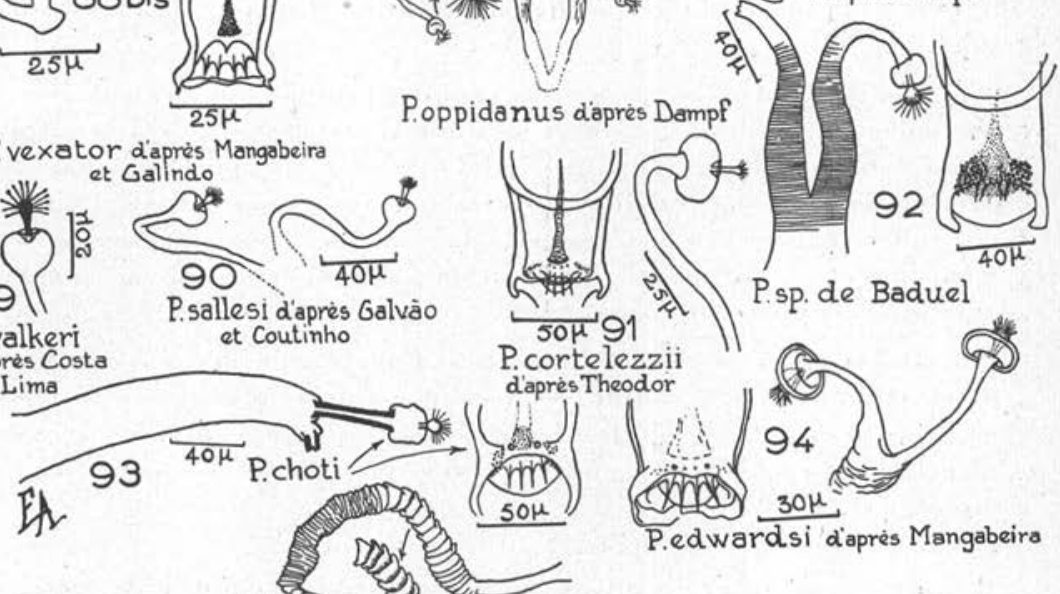

PLANCHE VII 